\title{
Precise Synthesis of Acrylate Copolymer via RAFT Polymerization and Its Application
}

\author{
Lei Li1,2, Jinxing Song1, Xin Yang ${ }^{1}$, Shengpeng Wang', Zuguang Li², Yingying Chen1, Aiyuan You ${ }^{1}$ \\ ${ }^{1}$ Transfar Zhilian Co., Ltd., Hangzhou, China \\ ${ }^{2}$ College of Chemical Engineering, Zhejiang University of Technology, Hangzhou, China \\ Email:1li0729@stu.suda.edu.cn
}

How to cite this paper: Li, L., Song, J.X., Yang, X., Wang, S.P., Li, Z.G., Chen, Y.Y. and You, A.Y. (2021) Precise Synthesis of Acrylate Copolymer via RAFT Polymerization and Its Application. Journal of Materials Science and Chemical Engineering, 9, 59-67.

https://doi.org/10.4236/msce.2021.96005

Received: March 22, 2021

Accepted: June 27, 2021

Published: June 30, 2021

\begin{abstract}
A series of different sequence structure containing stearyl acrylate (SA) and hydroxyethyl acrylate (HEA) copolymer were synthesized via reversible addition-fragmentation chain transfer (RAFT) polymerization. The crystallization property of PHEA- $b$-PSA, PHEA- $b$-PSA- $b$-PHEA, PSA- $b$-PHEA- $b$-PSA, PHEA-co-PSA, and PHEA-grad- $b$-PSA were characterized by Differential Scanning Calorimetry (DSC). The condensed structure of different sequence copolymers were investigated using small-angle X-ray scattering (SAXS). A series of copolymers with different sequence structure were coated on the surface of polyester fabric, which can be found that PHEA- $b$-PSA- $b$-PHEA block copolymer has good water repellency.
\end{abstract}

\section{Keywords}

RAFT Polymerization, Different Sequence Structure, Crystallization Property, Water Repellency

\section{Introduction}

Natural polymers form a great variety of self-assembled structures that are essential to their function. Extensive theoretical and experimental investigations have suggested that, beside the overall copolymer composition, the distribution of monomer units along polymer chains can be an important microstructural parameter for fine-tuning nano-morphologies, and thus the physical and functional properties of polymeric materials [1] [2]. The class of copolymers named random copolymer, block copolymer, and gradient copolymer. Among of them, block copolymer and gradient copolymer were difficult to synthesize using traditional radical polymerization. In recent years, with the development of controlled/living polymerization such as atom transfer radical polymerization (ATRP) 
[3] and reversible addition-fragmentation chain-transfer (RAFT), different sequence structure can be easily synthesized. In particular, RAFT polymerization enables the controlled polymerization of a wide range of functional monomers to form well-defined copolymers [4]. The acrylate copolymers with different sequence structure have different crystallization, thermal properties, mechanical properties and rheological properties. The different structural arrangement and the different movement modes of molecular chains, which also fully shows that the difference between properties is caused by the arrangement form of molecular chain segments and molecular movement [5] [6].

Acrylate copolymers containing a long-chain alkyl group such as stearyl acrylate (SA) and hydroxyethyl acrylate (HEA) are of great importance due to their potential applications as coating, water-proofing agent, and dispersant [7] [8] [9]. However, it is difficult to investigate the polymer morphology and distribution of polyester fabric, and structure-activity relationship between polymer structure and application properties.

Herein, we prepared random copolymer, block copolymer, and gradient copolymers with different sequence structure containing SA and HEA monomers using RAFT polymerization, as shown in Scheme 1 and Scheme 2. First, we prepared the targeted copolymers with controlling the same composition and ratio of monomers. Then, the chemical structure of the targeted copolymers were characterized by Fourier Transform Infra-Red (FT-IR) and Gel Permeation Chromatography (GPC). The crystallization property and aggregation structure of the targeted copolymers were investigated DSC and SAXS. Final, the water repellency of targeted copolymers were measured, which can be used to explain the internal relationship between structure and property and provide theoretical basis for practical production and application.

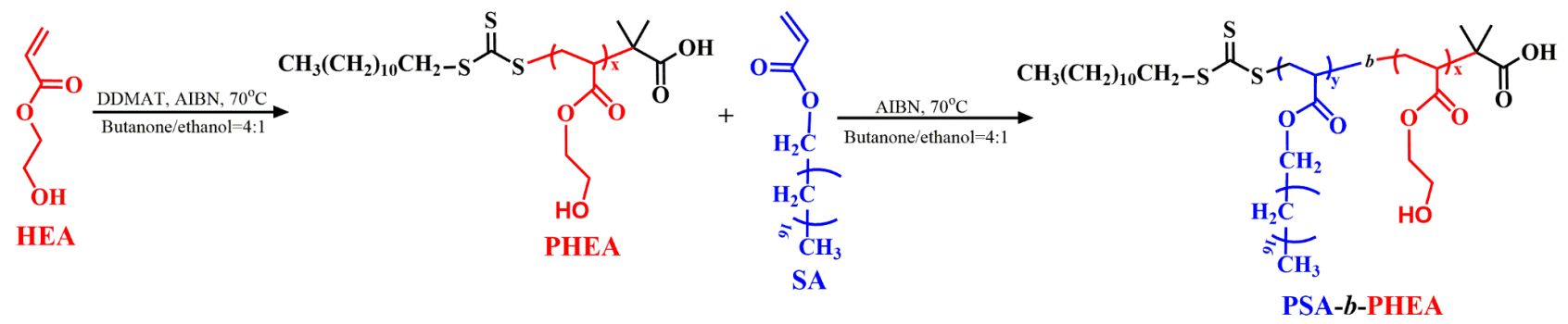

Scheme 1. Synthetic route of PHEA- $b$-PSA block copolymer.

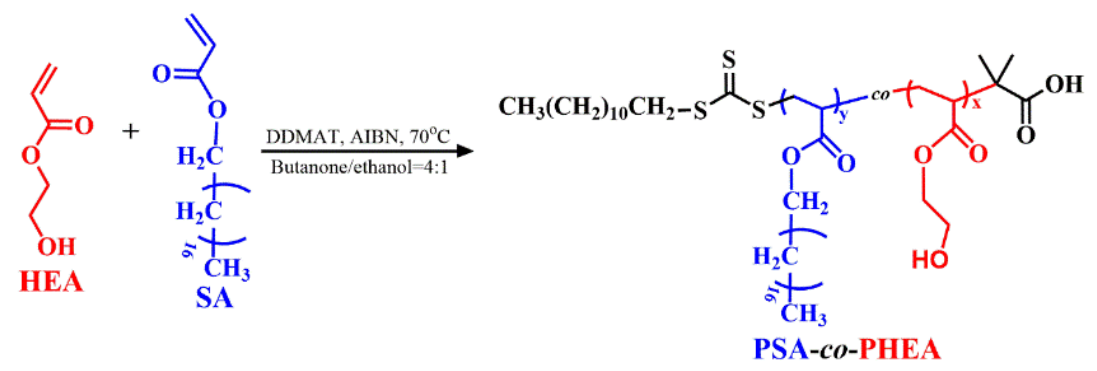

Scheme 2. Synthetic route of PHEA-co-PSA block copolymer. 


\section{Experimental}

\subsection{Materials}

The following agents were purchased and used without further purification: Stearyl acrylate (SA, 99\%, Sigma-Aldrich), hydroxyethyl acrylate (HEA, 98\%, Alfa Aesar), 2-(dodecylthiocarbonothioylthio)-2-methylpropionic acid (DDMAT, 98\%, Sigma-Aldrich). Azodiisobutyronitrile (AIBN, A. R., Enox) was purified by recrystallization from ethanol.

\subsection{Characterizations}

The number-average molecular weight $\left(\overline{M_{\mathrm{n}}}\right)$ and polydispersity index $(\nexists)$ of the targeted copolymers were analyzed with a gel permeation chromatography (GPC) instrument (Agilent 1100) using polystyrene as the standard and THF as the eluent. Differential scanning calorimetry was performed by a Perkin Elmer thermal analysis system equipped with a liquid-N2 low-temperature apparatus. The sample masses varied from 5.0 to $9.0 \mathrm{mg}$ were used for the DSC measurements, and all DSC experiments were carried out under a N2 atmosphere. The heating rate was $10^{\circ} \mathrm{C} / \mathrm{min}$ [10] [11]. Small angle X-ray scattering (SAXS, Shanghai Synchrotron Radiation Facility, BL16B1) with a wavelength of $1.24 \AA$, a sample-detector distance of $1860 \mathrm{~mm}$ and an exposed time of $300 \mathrm{~s}$ [12].

\subsection{Synthesis of PHEA- $b$-PSA Block Copolymer}

The PHEA- $b$-PSA copolymer was synthesized via RAFT polymerization. All magnetic stirring bars and glass ware used in the experiments were dried at $120^{\circ} \mathrm{C}$ for $24 \mathrm{~h}$ and cooled under vacuum to eliminate the moisture before use. Briefly, DDMAT (100 mg, $0.274 \mathrm{mmol}$ ), HEA (1.4 g, $12.1 \mathrm{mmol}$ ), and azobis(isobutyronitrile) (AIBN, $22.5 \mathrm{mg}, 0.137 \mathrm{mmol}$ ) were added into the flask together with $10 \mathrm{~mL}$ of butanone/ethanol $(\mathrm{v} / \mathrm{v}=4 / 1)$. After being degassed through three exhausting-refilling nitrogen cycles, the mixture was stirred under a nitrogen atmosphere at $70^{\circ} \mathrm{C}$ for $4 \mathrm{~h}$. Then, $10 \mathrm{~mL}$ of butanone/ethanol $(\mathrm{v} / \mathrm{v}=4 / 1)$ containing SA ( $4.2 \mathrm{~g}, 12.9 \mathrm{mmol})$ was added into the flask, and keep $70^{\circ} \mathrm{C}$ for $4 \mathrm{~h}$ under a nitrogen atmosphere. The flask was cooled to terminate the polymerization, the targeted copolymers was purified by removing the unreacted monomers. The final product PHEA- $b$-PSA copolymer was obtained by lyophilization method (Yield: 95.6\%).

\subsection{Synthesis of P(HEA-co-SA) Random Copolymer}

RAFT polymerization and the random copolymerization of HEA and SA were carried out in a round-bottomed flask with a silicone septum under an argon atmosphere. Briefly, DDMAT (100 mg, $0.274 \mathrm{mmol}$ ), HEA (1.4 g, $12.1 \mathrm{mmol}$ ), $\mathrm{SA}(4.2 \mathrm{~g}, 12.9 \mathrm{mmol}$ ) and azobis(isobutyronitrile) (AIBN, $22.5 \mathrm{mg}, 0.137 \mathrm{mmol}$ ) were added into the flask together with $20 \mathrm{~mL}$ of butanone/ethanol ( $\mathrm{v} / \mathrm{v}=4 / 1)$. After being degassed through three exhausting-refilling nitrogen cycles, the mixture was stirred under a nitrogen atmosphere at $70^{\circ} \mathrm{C}$ for $4 \mathrm{~h}$. The flask was 
cooled to terminate the polymerization, the targeted copolymers was purified by removing the unreacted monomers. The final product $\mathrm{P}$ (HEA-co-SA) random copolymer was obtained by lyophilization method. (Yield: 96.4\%)

\subsection{Synthesis of P (HEA-grad-SA) Gradient Copolymer}

The P (HEA-grad-SA) gradient copolymer was synthesized via RAFT polymerization. All magnetic stirring bars and glass ware used in the experiments were dried at $120^{\circ} \mathrm{C}$ for $24 \mathrm{~h}$ and cooled under vacuum to eliminate the moisture before use. Briefly, DDMAT (100 mg, $0.274 \mathrm{mmol}$ ), HEA (0.2 g, $1.7 \mathrm{mmol}$ ), SA (2.4 g, $7.4 \mathrm{mmol})$ and azobis(isobutyronitrile) (AIBN, $22.5 \mathrm{mg}, 0.137 \mathrm{mmol}$ ) were added into the flask together with $10 \mathrm{~mL}$ of butanone/ethanol (v/v = 4/1). After being degassed through three exhausting-refilling nitrogen cycles, the mixture was stirred under a nitrogen atmosphere at $70^{\circ} \mathrm{C}$ for $4 \mathrm{~h}$. Then, $10 \mathrm{~mL}$ butanone/ethanol of HEA $(0.4 \mathrm{~g}, 3.4 \mathrm{mmol})$ and SA $(1.2 \mathrm{~g}, 3.7 \mathrm{mmol})$ were added into the flask under a nitrogen atmosphere at $70^{\circ} \mathrm{C}$ for $4 \mathrm{~h}$. Finally, $10 \mathrm{~mL}$ butanone/ethanol of HEA $(0.8 \mathrm{~g}, 6.8 \mathrm{mmol})$ and SA $(0.6 \mathrm{~g}, 1.85 \mathrm{mmol})$ were added into the flask and stirred $4 \mathrm{~h}$ at $70^{\circ} \mathrm{C}$. The flask was cooled to terminate the polymerization, the targeted copolymers was purified by removing the unreacted monomers. The final product $\mathrm{P}(\mathrm{HEA}$-grad-SA) gradient copolymer was obtained. (Yield: $97.4 \%$ )

\section{Results and Discussion}

We synthesized PHEA- $b$-PSA, PHEA- $b$-PSA- $b$-PHEA, PSA- $b$-P $b$ HEA- $b$-PSA, $\mathrm{P}$ (HEA-co-SA), and P (HEA-grad-SA) via RAFT polymerization. The schematic diagram of targeted copolymers with different sequence structure are shown in

\section{Scheme 3.}

As shown in Figure 1, the chemical structure of PHEA- $b$-PSA, PHEA- $b$ PSA- $b$-PHEA, PSA- $b$-PHEA- $b$-PSA, P (HEA-co-SA), and P(HEA-grad-SA)

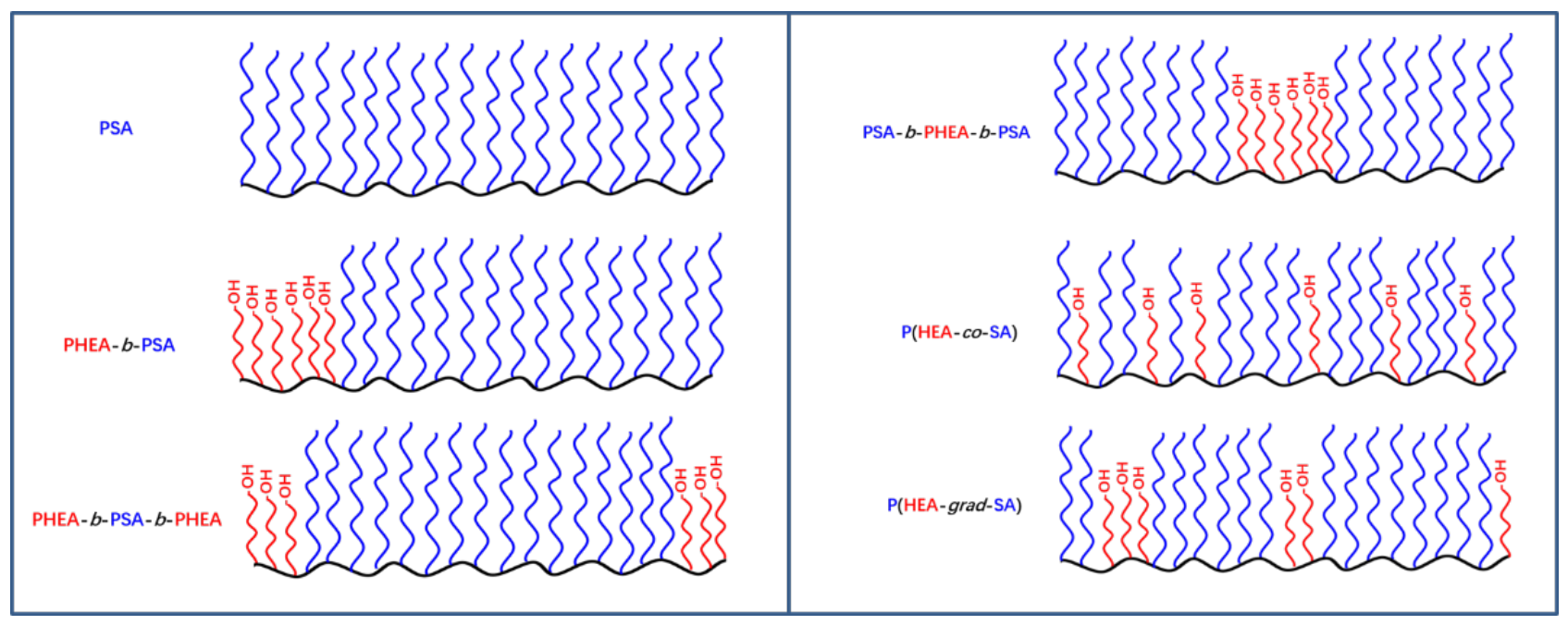

Scheme 3. The schematic diagram of targeted copolymers with different sequence structure. Abbreviated as PSA, PHEA- $b$-PSA, PHEA- $b$-PSA- $b$-PHEA, PSA- $b$-PHEA- $b$-PSA, P(HEA- $c o$-SA), and P(HEA-grad-SA). 
copolymers were characterized by FT-IR, which can demonstrate the characteristic peaks of the targeted copolymers, $3300 \mathrm{~cm}^{-1}, 2800 \mathrm{~cm}^{-1}$, and $1750 \mathrm{~cm}^{-1}$ are the stretching vibration peaks of $-\mathrm{OH},-\mathrm{CH}$, and $-\mathrm{C}=\mathrm{O}$ in PHEA- $b$-PSA, PHEA$b$-PSA- $b$-PHEA, PSA- $b$-PHEA- $b$-PSA, P(HEA-co-SA), and P (HEA-grad-SA) copolymers. We find that the targeted copolymers with different sequence structure have same characteristic absorption peak, this is to say, the differences of chemical structure could not be verified by FT-IR method.

Moreover, the molecular weight and molecular weight distribution of the different sequence structures were determined by GPC measurement in. The GPC trace of PSA (A), PHEA- $b$-PSA (B), PHEA- $b$-PSA- $b$-PHEA (C), PSA- $b$-PHEA$b$-PSA (D), $\mathrm{P}(\mathrm{HEA}-c o-\mathrm{SA})$ (E), and $\mathrm{P}$ (HEA-grad-SA) (F) copolymer in Figure 2 exhibited a unimodal peak, this means that random copolymers, block copolymers and gradient copolymers have been successfully prepared.

Moreover, recorded DSC curves of PSA, PHEA- $b$-PSA, PHEA- $b$-PSA- $b$-PHEA, PSA- $b$-PHEA- $b$-PSA, P (HEA-co-SA), and P(HEA-grad-SA) copolymers are presented in Figure 3. The crystallization have difference due to different SA distribution of the random copolymers, block copolymers, and gradient copolymers, we find that the crystallization temperatures of different sequence structure are $49.6^{\circ} \mathrm{C}, 39.5^{\circ} \mathrm{C}, 47^{\circ} \mathrm{C}, 37.3^{\circ} \mathrm{C}, 48.3^{\circ} \mathrm{C}$, and $45.9^{\circ} \mathrm{C}$, respectively. The more concentrated the SA segment distribution in the copolymer, the crystallization temperature increases. Hence, we can control the crystallization of copolymers through changing the degree of aggregation of crystalline fragments.

Water repellency of polyester fabric was evaluated according to AATCC 22-2010 "Water Repellency: Spray Test", as shown in Table 1. L0 represents the initial waterproof score and L5 represents the waterproof score after washing for five times. We find that the different sequence structure with same molecular weight possess the different waterproof score, the water repellency of PSA,

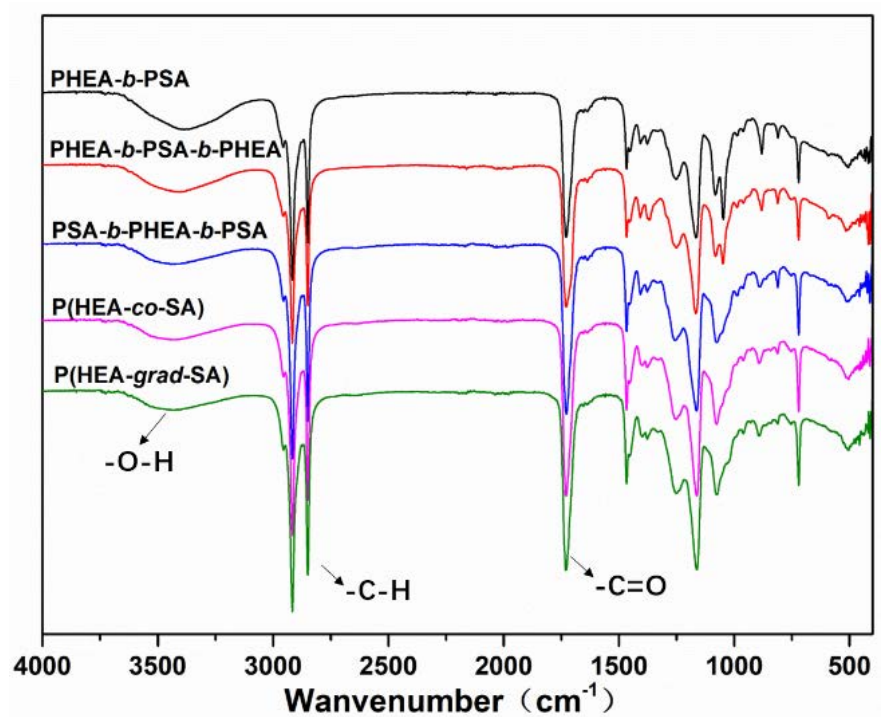

Figure 1. FT-IR spectra of PHEA- $b$-PSA, PHEA- $b$-PSA- $b$-PHEA, PSA- $b$-PHEA- $b$-PSA, P (HEA-co-SA), and P (HEA-grad-SA) copolymer. 

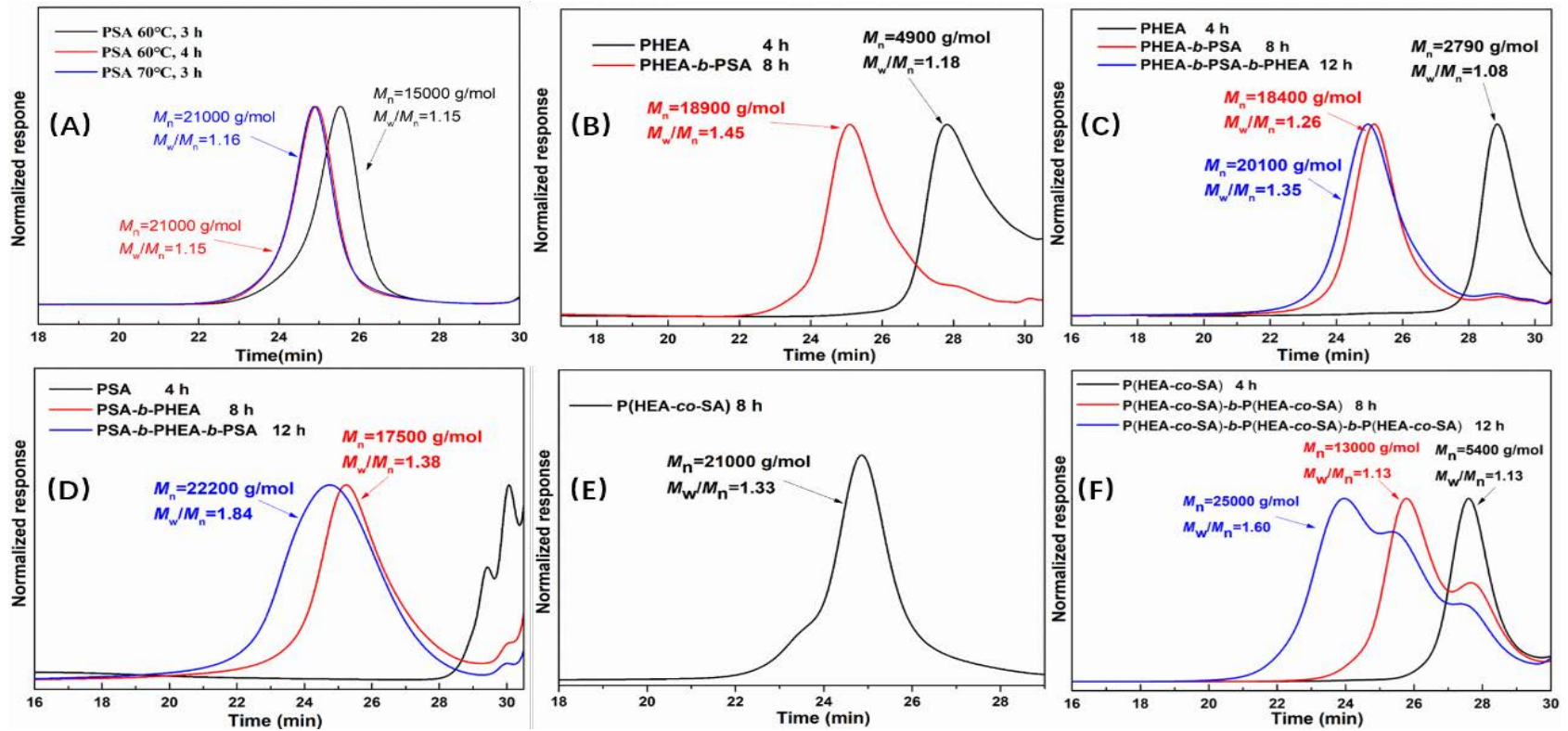

Figure 2. GPC traces curves of PSA (A), PHEA- $b$-PSA (B), PHEA- $b$-PSA- $b$-PHEA (C), PSA- $b$-PHEA- $b$-PSA (D), P(HEA- $c$-SA) (E), and P(HEA-grad-SA) (F) copolymer.
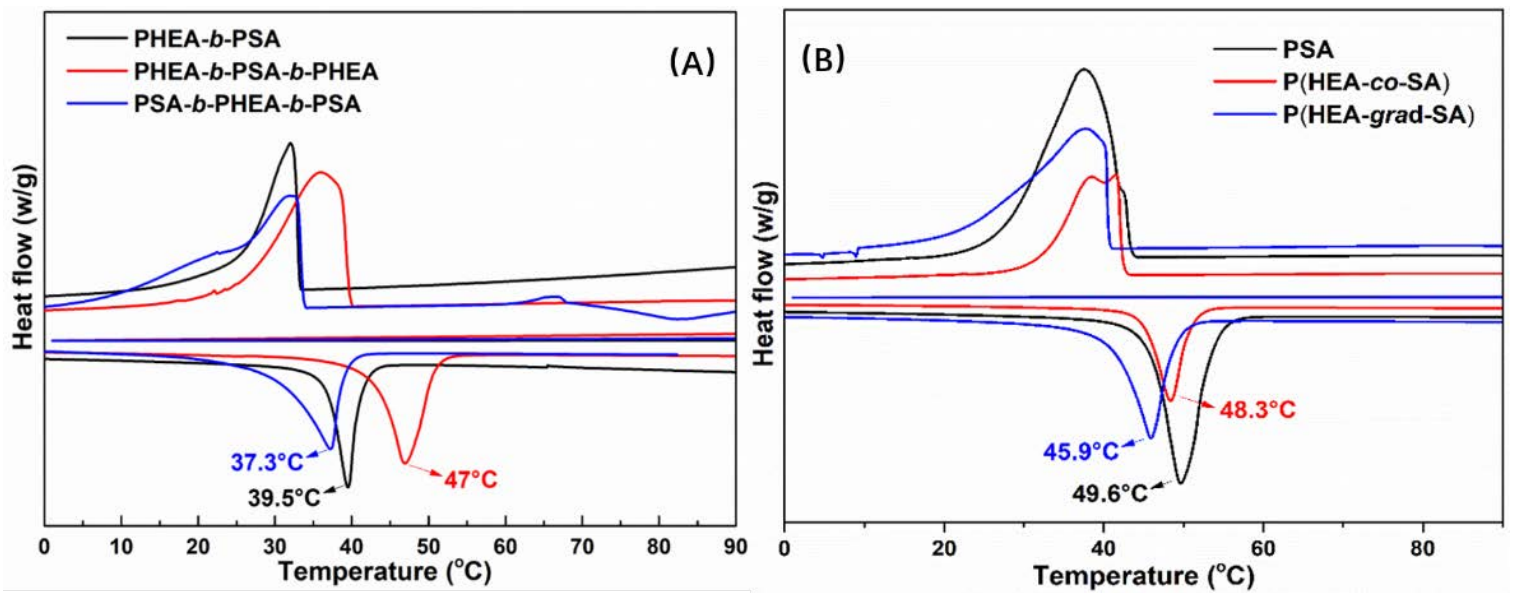

Figure 3. DSC scans for PSA, PHEA- $b$-PSA, PHEA- $b$-PSA- $b$-PHEA, PSA- $b$-PHEA- $b$-PSA, P (HEA-co-SA), and P (HEAgrad-SA) copolymer.

Table 1. Water repellency of PHEA- $b$-PSA, PHEA- $b$-PSA- $b$-PHEA, PSA- $b$-PHEA- $b$ PSA, P(HEA-co-SA), and P(HEA-grad-SA), and PSA.

\begin{tabular}{cccccc}
\hline \multirow{2}{*}{ Sample } & $\left(\overline{M_{\mathbf{n}}}, \mathbf{g} / \mathrm{mol}\right)$ & \multicolumn{2}{c}{ No cross-linking agent } & \multicolumn{2}{c}{ Cross-linking agent } \\
\cline { 3 - 6 } & & L0 & L5 & L0 & L5 \\
\hline PHEA- $b$-PSA & 19,000 & 50 & $0-50$ & $80+$ & 95 \\
PHEA- $b$-PSA- $b$-PHEA & 20,100 & $50-$ & $0-50$ & $80+$ & 100 \\
PSA- $b$-PHEA- $b$-PSA & 22,000 & $70+$ & $0-50$ & 80 & 95 \\
P(HEA-co-SA $)$ & 21,000 & 70 & $0-50$ & 75 & $80+$ \\
P(HEA-grad-SA) & 20,400 & 60 & $0-50$ & $80+$ & 95 \\
PSA & 15,000 & 70 & $0-50$ & $70-$ & 0 \\
\hline
\end{tabular}




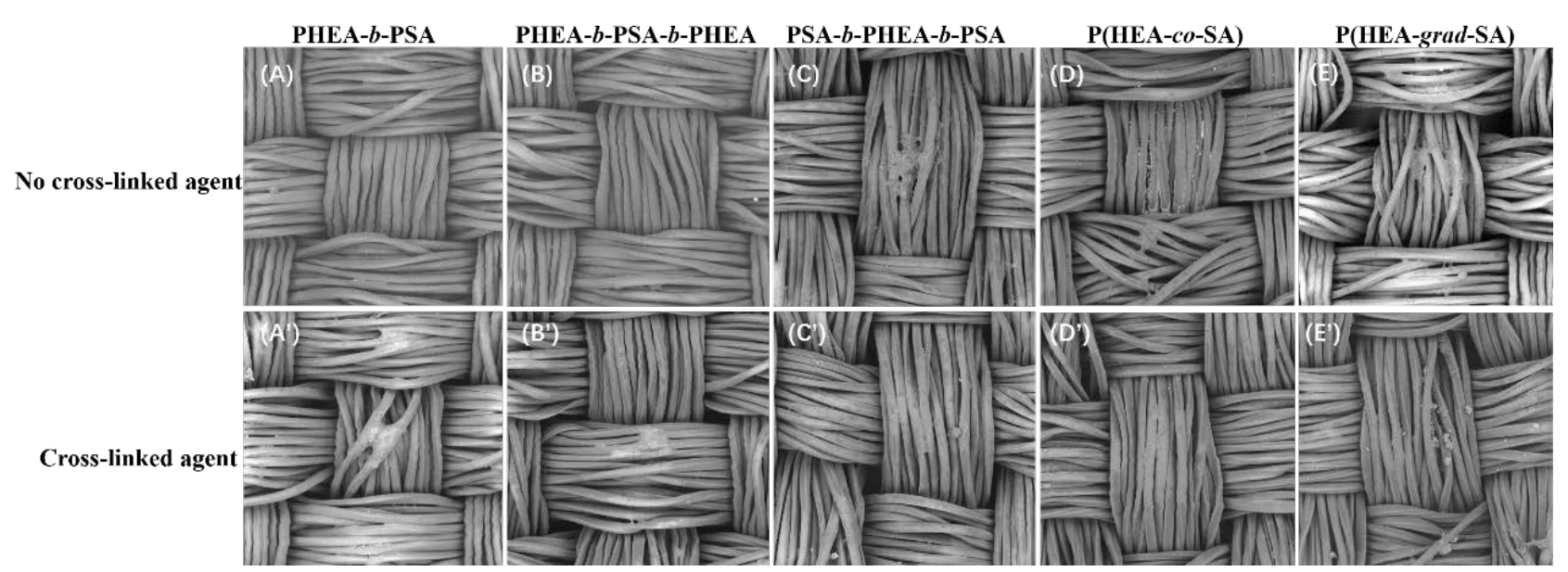

Figure 4. SEM images of PHEA- $b$-PSA, PHEA- $b$-PSA- $b$-PHEA, PSA- $b$-PHEA- $b$-PSA, P (HEA-co-SA), and P (HEA-grad-SA) copolymer coating polyester fabric. ((A), (B), (C), (D), and (E) represent the absence of a cross-linker. (A'), (B'), (C'), (D'), and (E') represent the addition of a cross-linker).

PHEA- $b$-PSA, PHEA- $b$-PSA- $b$-PHEA, PSA- $b$-PHEA- $b$-PSA, P(HEA- $c o$-SA), and $\mathrm{P}$ (HEA-grad-SA) copolymers could be improved with adding the crosslinked agents when the different copolymers finishing on polyester fabrics were performed. Among of them, the PHEA- $b$-PSA- $b$-PHEA have better water repellency. This is due to the HEA segments of PHEA- $b$-PSA- $b$-PHEA not only could act as a cross-linking role, but also fix the SA segment to come true the excellent washing resistance.

SEM images of the different copolymers coated polyester fabric were illustrated in Figure 4. Among of them, we find that the fabric with no cross-linked agents showed cylindrical structure and small amounts of copolymers aggregation in Figures 4(A)-(E). After the coated polyester fabric with adding the cross-linked agents, an evidently uneven film was markedly generated on the surface of the polyester fiber in Figures 4(A')-(E'). The PHEA- $b$-PSA- $b$-PHEA block copolymers coated polyester fabric possess uniform circular distribution at high baking temperature, which confirmed that PHEA- $b$-PSA- $b$-PHEA had been successfully finished onto the surface of polyester fabric.

As we all know, the intensity of SAXS is dependent on the electron density difference between the phases of the system. Lorentz-corrected intensity $\mathrm{Iq}^{2}$ is a function of the scatter vector $q$ under different shear temperatures along and perpendicular to the shear direction. From Figures 5(A)-(C), we can see that two scattering peaks appear in the block copolymers. As shown Figure 5(D) and Figure $5(\mathrm{E})$, the one peaks appear in the random and gradient copolymers, which indicate that the different copolymers have a great difference in the electron density of the system, this is due to the segmentation degree and crystallization of SA chain.

\section{Conclusion}

The random copolymer, block copolymer, and gradient copolymers with different 

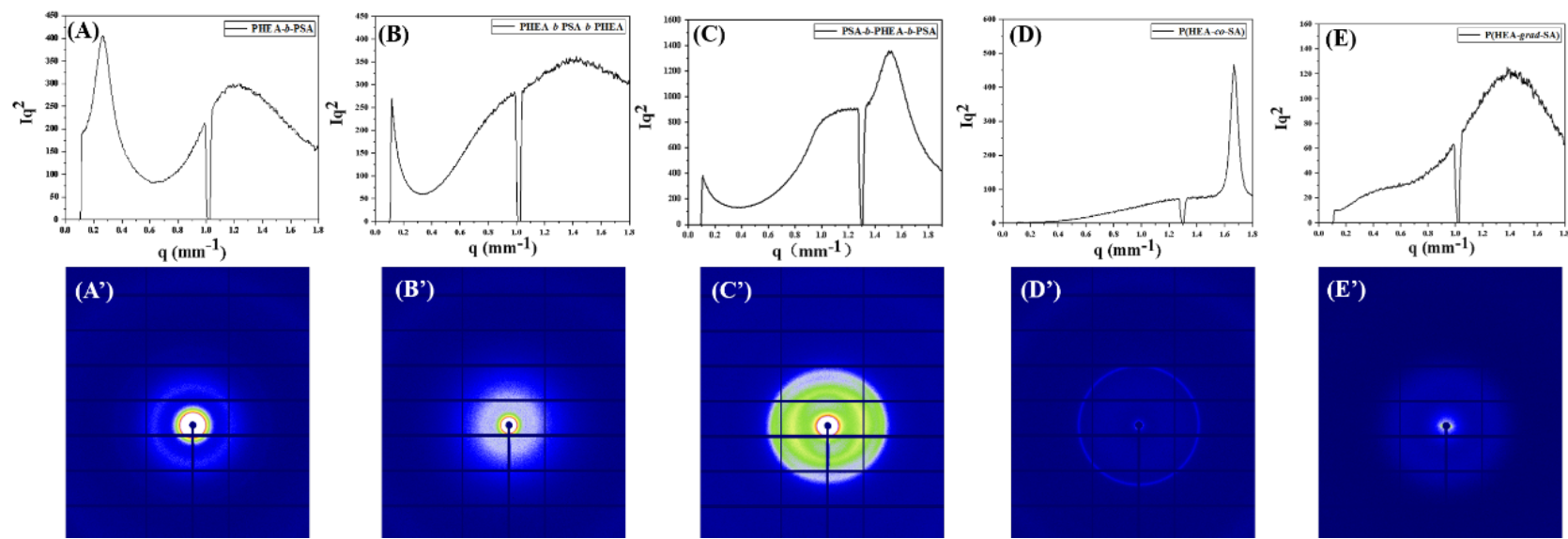

Figure 5. Iq ${ }^{2}$-q curves collected by SAXS and 2D SAXS patterns of PHEA- $b$-PSA ((A) and (A')), PHEA- $b$-PSA- $b$-PHEA ((B) and (B')), PSA- $b$-PHEA- $b$-PSA ((C) and (C')), P(HEA-co-SA) ((D) and (D')), and P(HEA-grad-SA) ((E) and (E')).

sequence structure containing SA and HEA monomers have been synthesized via RAFT polymerization. The molecular weight and molecular weight distribution of the different copolymers have verified. The crystallization temperatures of different sequence structure are $49.6^{\circ} \mathrm{C}, 39.5^{\circ} \mathrm{C}, 47^{\circ} \mathrm{C}, 37.3^{\circ} \mathrm{C}, 48.3^{\circ} \mathrm{C}$, and $45.9^{\circ} \mathrm{C}$, respectively. What is more important, the PHEA- $b$-PSA- $b$-PHEA possess good water repellency due to the uniform circular distribution at high baking temperature with adding the cross-linked agents. Moreover, the different copolymers have difference in the electron density due to the segmentation degree and crystallization of SA chain.

\section{Acknowledgements}

Dr. Lei Li would like to thank the financial support from the Postdoctoral Research Program of Zhejiang Province (ZJ2020058). Dr. Lei Li thanks Ms. Shuhui Ding for her help in revising the manuscript.

\section{Conflicts of Interest}

The authors declare no conflicts of interest regarding the publication of this paper.

\section{References}

[1] Zhang, J.L., Farias-Mancilla, B., Kulai, I., Hoeppener, S., Lonetti, B., Prévost, S., Ulbrich, J., Destarac, M., Colombani, O., Schubert, U.S., Guerrero-Sanchez, C. and Harrisson, S. (2021) Effect of Hydrophilic Monomer Distribution on Self-Assembly of a pH-Responsive Copolymer: Spheres, Worms and Vesicles from a Single Copolymer Composition. Angew. Chem. Int. Ed., 60, 4925-4930. https://doi.org/10.1002/anie.202010501

[2] Alama, M.M., Peng, H., Jack, K.S., Hill, D.J.T. and Whittaker, A.K. (2020) Synthesis of 4-Acetoxystyrene-t-Butyl Acrylate Statistical, Block and Gradient Copolymers, and the Effect of the Structure of Copolymers on Their Properties. Eur Polym. J., 134, 109772. https://doi.org/10.1016/j.eurpolymj.2020.109772

[3] Whitfield, R., Anastasaki, A., Nikolaou, V., Jones, G.R., Engelis, N.G., Discekici, 
E.H., Fleischmann, C., Willenbacher, J., Hawker, C.J. and Haddleton, D.M. (2017) Universal Conditions for the Controlled Polymerization of Acrylates, Methacrylates, and Styrene via Cu(0)-RDRP. J. Am. Chem. Soc., 139, 1003-1010.

https://doi.org/10.1021/jacs.6b11783

[4] Messerschmidt, M., Komber, H., Häußler, L., Hanzelmann, C., Stamm, M., Raether, B., Silva, O.C. and Uhlmann, P. (2013) Amphiphilic ABC Triblock Copolymers Tailored via RAFT Polymerization as Textile Surface Modifiers with Dual-Action Properties. Macromolecules, 46, 2616-2627. https://doi.org/10.1021/ma302471q

[5] Yamano, T., Higashi, N. and Koga, T. (2020) Unique Self-Assembly of SequenceControlled Amino Acid Derived Vinyl Polymer with Gradient Thermoresponsiveness along a Chain. Langmuir, 36, 6550-6556. https://doi.org/10.1021/acs.langmuir.0c01036

[6] Brotherton, E.E., Hatton, F.L., Cockram, A.A., Derry, M.J., Czajka, A., Cornel, E.J., Topham, P.D., Mykhaylyk, O.O. and Armes, S.P. (2019) In Situ Small-Angle X-Ray Scattering Studies during Reversible Addition-Fragmentation Chain Transfer Aqueous Emulsion Polymerization. J. Am. Chem. Soc., 141, 13664-13675. https://doi.org/10.1021/jacs.9b06788

[7] Zhao, J., Zhu, W.X., Wang, X.F., Liu, L.F., Yu, J.Y. and Ding, B. (2020) FluorineFree Waterborne Coating for Environmentally Friendly, Robustly Water-Resistant, and Highly Breathable Fibrous Textiles. ACS Nano, 14, 1045-1054. https://doi.org/10.1021/acsnano.9b08595

[8] Messerschmidt, M., Janke, A., Simon, F., Hanzelmann, C., Riske, T., Stamm, M., Raether, B., Silva, O.C. and Uhlmann, P. (2018) Fluorocarbon-Free Dual-Action Textile Finishes Based on Covalently Attached Thermoresponsive Block Copolymer Brush Coatings. ACS Appl. Mater. Interfaces, 10, 40088-40099. https://doi.org/10.1021/acsami.8b11448

[9] Smith, A.A.A., Autzen, H.E., Laursen, T., Wu, V., Yen, M., Hall, A., Hansen, S.D., Cheng, Y.F. and Xu, T. (2017) Controlling Styrene Maleic Acid Lipid Particles through RAFT. Biomacromolecules, 18, 3706-3713. https://doi.org/10.1021/acs.biomac.7b01136

[10] Stoclet, G., Seguela, R., Lefebvre, J.M. and Rochas, C. (2010) New Insights on the Strain-Induced Mesophase of Poly(d,l-lactide): In Situ WAXS and DSC Study of the Thermo-Mechanical Stability. Macromolecules, 43, 7228-7237. https://doi.org/10.1021/ma101430c

[11] Gao, X.Z., Wang, L.Y., Luo, H., Zou, Q., Feng, N.D. and Feng, J.W. (2010) Crystalline Phases in Ethylene Copolymers Studied by Solid-State NMR and DSC. Macromolecules, 43, 5713-5722. https://doi.org/10.1021/ma1000578

[12] Xie, K.Y., Shen, J.Q., Ye, L.J., Liu, Z.Y. and Li, Y.J. (2019) Increased gt Conformer Contents of PLLA Molecular Chains Induced by Li-TFSI in Melt: Another Route to Promote PLLA Crystallization. Macromolecules, 52, 7065-7072. https://doi.org/10.1021/acs.macromol.9b01188 